\title{
RECURSOS HÍDRICOS: CALIDAD Y PROCESOS DE GESTIÓN EN LA REGIÓN PAMPEANA ARGENTINA
}

\author{
Corina Iris Rodríguez \\ Guillermina Paula Jacinto (D) ${ }^{2}$
}

Recibido el 6 de noviembre de 2015, aprobado el 2 de mayo de 2016 y actualizado el

12 de diciembre de 2016

DOI: 10.17151/luaz.2017.44.5

\section{RESUMEN}

El objetivo es analizar la situación de los recursos hídricos subterráneos en un área del sudeste de la región pampeana argentina. A través de estudios de caso, se presenta un diagnóstico de la calidad del agua para consumo humano en dos pueblos rurales de la Provincia de Buenos Aires, mostrando los modos de uso, los procesos de gestión y los impactos potenciales que las transformaciones socioterritoriales por las que atraviesan aquellos pueblos podrían generar sobre los recursos hídricos. Las pequeñas localidades -María Ignacia y Gardey- funcionan como centros que prestan servicios básicos al entorno rural. Investigaciones recientes ponen de manifiesto que los vínculos que mantienen con la ciudad cabecera de Tandil, han activado en ellos un conjunto de transformaciones cuyo origen y dinámica no obedecen únicamente a su rol de prestación de servicios rurales, sino que se entroncan con las demandas de espacios y actores urbanos. La expansión residencial y la presencia de nuevos usos de suelo como los emprendimientos recreativo-turísticos inducen presión sobre los recursos naturales en general, y sobre la disponibilidad y calidad de agua para consumo, en particular, abriendo nuevos desafíos para los procesos de gestión y gobernanza del agua a escala local.

\section{PALABRAS CLAVE}

Agua subterránea, calidad, gestión, pueblos rurales.

\section{WATER RESOURCES: QUALITY AND MANAGEMENT PROCESSES IN THE PAMPEAN REGION OF ARGENTINA}

\section{ABSTRACT}

This work is aimed to analyze the groundwater resources in the southeast area of the pampean region in Argentina. A diagnosis of water quality in two rural villages of Buenos Aires province is presented through study cases. This work shows the ways of water use, the management processes and the potential impacts that territorial transformation could cause on water resources. The small rural villages -María Ignacia and Gardey - function as centers which provide basic services to the rural scope. Recent research works demonstrated that the links between these villages and the head city of Tandil have activated a set of territorial transformations whose origin and dynamic are not only related to the role of bringing rural services, but also are based on the demand of urban activities and stakeholders. The residential expansion and the presence of new land uses, as recreational and tourist activities, make pressure on natural resources and also press on water availability and quality for human consumption. These transformations generate new challenges for water management processes and its governance at local scope.

\section{KEY WORDS}


Groundwater, quality, management, rural villages.

\section{INTRODUCCIÓN}

El interés por el estudio de los recursos hídricos para consumo humano en localidades rurales de la región pampeana ha ocupado un lugar marginal en los programas de investigación sobre utilización y manejo de recursos. La disponibilidad y acceso al agua para el desarrollo de la vida y de las actividades productivas nunca fueron percibidos como problemas en la región más rica de la Argentina, donde la profundidad de los niveles freáticos y la calidad de los acuíferos permitieron su explotación y consumo sin mayores dificultades, procurado incluso individualmente, en ausencia de redes de aprovisionamiento. Esto se relaciona directamente con la escasez relativa de población rural que podría verse involucrada en problemas de abastecimiento o afectación de la calidad de los recursos hídricos, en una región donde más del $97 \%$ de la población es urbana, residiendo en aglomeraciones que superan los 2000 habitantes.

En tanto el patrón de asentamientos que ha organizado el territorio nacional y a la región pampeana durante el siglo XX se ha caracterizado históricamente por la primacía metropolitana de Buenos Aires y el crecimiento sostenido de ciudades medias, ambos procesos fueron el correlato del decrecimiento sostenido de la población rural, principalmente de la población dispersa en "campo abierto" (Vapñarsky y Gorojovsky, 1990). En este escenario, la reducción de la población rural, activada por el avance de las relaciones capitalistas de producción, con promesa de empleo industrial y mejores condiciones de vida urbana, comprometieron la supervivencia de las localidades rurales. Tanto es así que durante la década de los 90, la disolución de los tejidos socio-productivos desencadenada por la penetración del capital financiero en los espacios rurales, junto con la desarticulación del sistema ferroviario de cargas que explicaba la existencia de buena parte de los pueblos, terminó de perfilar el cuadro de agonía que alcanzó a la mayor parte de los pueblos rurales en esta región.

Mientras en la mayoría de los casos los macroprocesos homogeneizantes condujeron a la fragilización y a la desaparición de las localidades rurales, iniciados el siglo XXI, algunas indagaciones empíricas comenzaron a mostrar su revitalización demográfica, e incluso económica (Sili, 2005 y 2010). Aún bajo procesos hegemónicos de concentración de la tierra, mecanización agrícola y expulsión de actores, comenzó a identificarse la presencia y expansión de nuevos usos, no agrícolas, impulsados por las demandas de sociedades urbanas. El crecimiento de la segunda residencia y del empleo agrario a tiempo parcial, así como la instalación de nuevos pobladores que fijaban residencia permanente en el espacio rural y mantenían su empleo urbano, daban cuenta de la revitalización de algunos pueblos rurales. Complementariamente, el mejoramiento de las condiciones de comunicación, el desarrollo de la movilidad individual, el acceso al mercado de tierras y la calidad socio-ambiental, reactivaron una vinculación de carácter selectivo entre algunas localidades y las ciudades próximas de mayor tamaño en la región pampeana (Jacinto et al., 2014).

De esta manera, los pueblos rurales ganaron visibilidad en numerosos programas de investigación, abordándolos como territorios donde explorar no sólo los cambios en la dinámica local o en su rol funcional sino, y fundamentalmente, los impactos asociados 
a su inscripción en una región donde se profundizaba el proceso de agriculturización con alta presión y compromiso ambiental sobre los recursos.

Alcanzada por el proceso de homogeneización productiva, el área de estudio ha experimentado cambios en los usos del suelo rural marcados por el repliegue de la ganadería, la sustitución de cultivos y cambios en la participación relativa de cada uno de ellos en la matriz productiva local. Desde mediados de la década de 1990, la expansión agrícola implicó aumento de productividad y de la superficie sembrada, impulsados por la incorporación de soja como líder entre los cultivos, por el crecimiento de la demanda internacional y por la presencia de nuevos actores como los pools de siembra y los contratistas de producción. En las últimas dos décadas, la competencia con la agricultura provocó también profundos cambios en la localización de la ganadería vacuna, desplazándola hacia zonas marginales y sustituyendo la ganadería extensiva por sistemas intensivos de producción (feed lot).

Las modalidades e impactos relacionados con nuevas formas de apropiación de los recursos se constituyen así en puntos de interés, en tanto los procesos en marcha (intensificación agrícola, despliegue de redes agroindustriales, expansión residencial y demanda de servicios) activan tensiones en torno al uso y manejo de los recursos en general, y de los recursos hídricos en particular. Tradicionales y nuevas actividades, antiguos residentes y nuevos usuarios, afectan las interrelaciones y demandas sobre la disponibilidad, cantidad y calidad del agua para consumo y desarrollo de actividades. Ello redunda en la necesidad de evaluar la situación presente tanto como de prever un conjunto de acciones que involucren expectativas cuali-cuantitativas de consumo, solidarias con una gestión integrada y sustentable de los recursos.

\section{USO Y MANEJO DE LOS RECURSOS HÍDRICOS EN LOCALIDADES RURALES DE TANDIL}

En la encrucijada de procesos, cuyos actores y lógicas de acción se despliegan en contextos multiescalares, además de centros de servicio para las poblaciones y actividades rurales, los pueblos rurales pampeanos albergan funciones vinculadas con la imagen de campagne-ressource (funciones productivas), campagne-cadre de vie(funciones residenciales y turístico-recreativas) y campagne-nature (funciones naturales y servicios ecosistémicos), que actúan como factores de revitalización y vectores de reapropiación de lo rural por lo urbano (Perrier-Cornet, 2002).

Las ventajas de proximidad y accesibilidad a través de caminos asfaltados, que garantizan la conexión fluida con sus cabeceras departamentales, han reposicionado a numerosas localidades rurales como espacios de residencia permanente. Esto se potencia fundamentalmente porque la presión sobre los mercados inmobiliarios urbanos y el sostenido incremento del precio del suelo urbanizable en las ciudades próximas, dificultan las posibilidades de acceso a la vivienda. En este contexto, los pueblos rurales son elegidos para fijar residencia permanente por los menores precios del suelo, inaugurándose en ellos un acelerado proceso de valorización de tierras vacantes y de bienes inmuebles durante la última década.

La expansión residencial se evidencia en el aumento del número de viviendas que ocupan terrenos vacantes, y su proyección se sostiene a partir de la oferta sin cesar de tierras incorporadas al mercado, con asistencia del gobierno municipal en la regularización dominial y con ampliación de la planta de viviendas en construcción financiada por programas nacionales como PROCREAR (Programa de Crédito Argentino) (Villalba, 2015). 
En simultáneo al proceso de expansión urbana, se incrementa la necesidad de construcción de infraestructuras y la dotación de servicios, principalmente de agua, gas natural y cloacas. El punto más crítico lo constituye el alto costo de construcción de las redes demandadas y el bajo número de usuarios entre los cuales prorratear la inversión. En un escenario de falta de autonomía -cuando el servicio es proporcionado por terceros-, y de estrangulamiento presupuestario -cuando el servicio es brindado por las administraciones municipales-, las localidades rurales ven afectada la provisión de servicios en términos cualitativos y cuantitativos.

Cabe comentar que en la provincia de Buenos Aires, donde se localiza el partido de Tandil, los recursos hídricos son considerados propiedad del Estado provincial y son regulados por la Autoridad del Agua. Este organismo es de tipo centralizado y su sede está ubicada en la capital provincial. El Código de Aguas de la provincia de Buenos Aires, Ley $\mathrm{N}^{\circ} 12.257$, establece que dicha autoridad supervisa todas las obras de captación, uso y evacuación del agua, y otorga concesiones para el abastecimiento del servicio de agua potable a distintos entes como cooperativas y municipios. Además, exige que los usuarios particulares deban solicitar un permiso de perforación para extraer agua subterránea.

En relación a la provisión de servicios de agua, si bien existe el servicio de abastecimiento por red, numerosas viviendas no se encuentran conectadas y las localizadas en la periferia de los ejidos no están abastecidas. La utilización de pozos particulares permanece como una estrategia de las viviendas particulares; muchos de ellos evidencian deficiencias de diseño y de protección sanitaria, así como algunos casos puntuales de contaminación (Rodríguez, 2014). Esto último se relaciona principalmente con la ausencia de sistemas de recolección y tratamiento de efluentes cloacales, y la existencia de pozos sépticos y letrinas próximas a las bocas de consumo, así como la presencia de potenciales focos contaminantes como la disposición de insumos para el agro y de residuos sólidos urbanos. Ello da cuenta de la necesidad de interrogarse en torno a la calidad y cantidad de agua disponible para sostener la calidad de vida en los pueblos rurales del sudeste de la región pampeana.

\section{Casos de estudio: María Ignacia y Gardey}

María Ignacia (2000 habitantes) y Gardey (640 habitantes) son los dos pueblos rurales de mayor tamaño del Partido de Tandil. Localizados en la subcuenca del arroyo Chapaleofú Chico (Figura 1), ambos núcleos funcionan como centros de servicios rurales de primera categoría que tradicionalmente han prestado servicios al entorno rural (Municipalidad de Tandil, 2005).

En ambos casos la única fuente de abastecimiento de agua para todos los usos es la subterránea, proveniente del acuífero libre. 


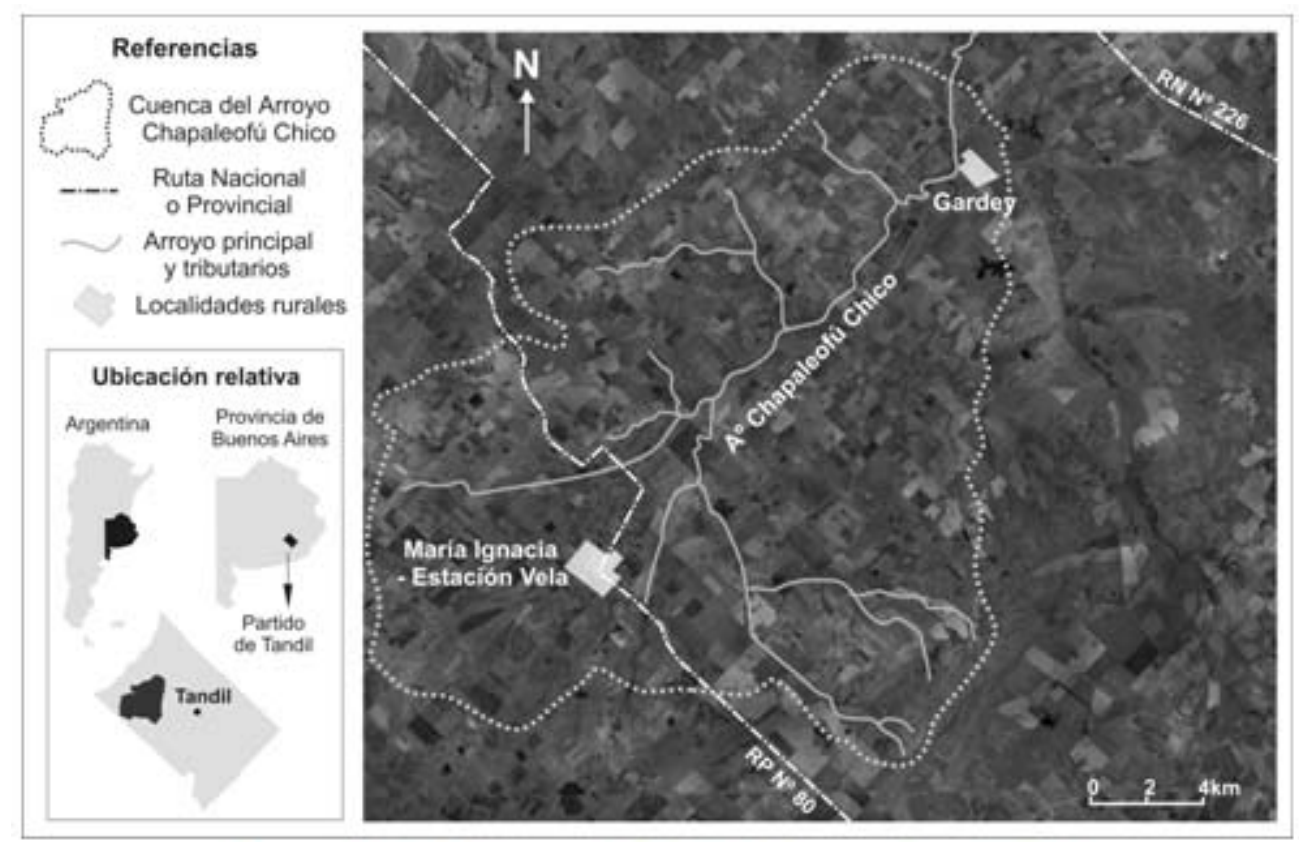

Figura 1. Localización de los casos de estudio.

Como lo indicáramos más arriba, la problemática vinculada al manejo de los recursos hídricos en ambos asentamientos de rango menor (ARM), se presenta como un aspecto sustancial en relación a las transformaciones territoriales producidas durante la última década (Jacinto, 2011).

\section{ABORDAJE METODOLÓGICO}

En cada caso estudiado se indagó sobre los modos de uso del agua subterránea por parte de los habitantes y se realizaron entrevistas a informantes clave para conocer la gestión institucional de los recursos hídricos a escala local.

Asimismo, se efectuaron censos de perforaciones, con la finalidad de conocer la dinámica del acuífero y evaluar la calidad del agua para consumo humano, tanto en sus aspectos físico-químicos como microbiológicos. En cada punto de extracción de agua relevado, se midieron los niveles freáticos, se tomaron muestras de agua, se efectuaron encuestas a los pobladores sobre sus modos de abastecimiento y uso del agua, y se registró información sobre las características constructivas de las perforaciones, existencia de focos contaminantes cercanos a las mismas, sistemas de disposición de efluentes, antecedentes de contaminación del agua o enfermedades de origen hídrico.

\section{RESULTADOS Y DISCUSIÓN}

\section{María Ignacia}

\section{a. Modos de uso y gestión del agua subterránea}


El suministro de agua proviene de dos perforaciones que explotan el acuífero freático: una construida en 1980 de $59 \mathrm{~m}$ de profundidad, la otra de 2007, alcanza los $63 \mathrm{~m}$. El abastecimiento de agua potable alcanza aproximadamente al $80 \%$ de la población (500 bocas de abastecimiento) y el consumo poblacional medio es de $400 \mathrm{~m} 3 /$ día (Rodríguez et al., 2013).

Los efluentes domiciliarios son vertidos principalmente en pozos absorbentes y en letrinas, afectando la calidad del agua del acuífero freático, fuente de extracción de agua para la población local. Se encuentra en progreso el proyecto de construcción del sistema cloacal para el tratamiento de efluentes domiciliarios, dimensionado para unos 2.300-2.500 habitantes, aunque la proyección de crecimiento del $10 \%$ del asentamiento de la última década (2001-2010), indica que se superaría la capacidad operativa de la planta cloacal en los próximos 15 a 20 años.

Desde 1980, el servicio de provisión de agua potable es brindado por una cooperativa que se encarga de la extracción de agua, sometiéndola a un proceso de cloración automática para garantizar la desinfección previa al almacenamiento y la distribución. Efectúa análisis periódicos de la calidad el recurso, cuyos resultados más recientes indican aptitud para el consumo humano.

La cooperativa funciona bajo la coordinación del Servicio Provincial de Agua Potable y Saneamiento Rural (SPAR). El municipio de Tandil (gobierno local) no tiene injerencia en el servicio de agua potable, por lo que en casos de viviendas con problemas de calidad del agua en sus pozos particulares, no existe una medida preventiva o correctiva a escala local.

Tanto en el ejido principal como a proximidad del asentamiento, los pobladores utilizan simultáneamente perforaciones particulares para la obtención del recurso. La mayor parte de los sistemas de extracción particulares son perforaciones someras, aunque también existen pozos cavados, de construcción antigua, cuyas profundidades oscilan entre los 5 y $33 \mathrm{~m}$, a excepción de las pertenecientes a la cooperativa. Para la extracción de agua, se utilizan principalmente bombas de mano, seguidas por bombeadores automáticos ubicados en superficie y molinos. Es muy reciente la colocación de bombas sumergibles que sólo se encontraron en la cooperativa y en una vivienda particular.

En general se presentan importantes deficiencias en el diseño, construcción y protección sanitaria de las perforaciones, siendo escasas aquellas que poseen cañería de encamisado y sellado de las paredes del pozo. Además, la gran mayoría no cuenta con cobertura superficial adecuada para impedir la contaminación, llegando incluso en muchos casos a la ausencia de tapa o cubierta.

La opción de mantener en uso la perforación o pozo domiciliario, prefiriendo en algunos casos no conectarse a la red de agua potable, se relaciona por un lado con factores económicos (el uso es medido y arancelado según intervalos de consumo), pero fundamentalmente porque a los usuarios les resulta más agradable el sabor del agua de pozo y confían en su calidad.

Con respecto a la presencia de focos contaminantes cercanos, se detectó que en el $81 \%$ de los casos relevados el distanciamiento entre los pozos absorbentes y las perforaciones es menor a $15 \mathrm{~m}$ (Rodríguez, 2014). Al considerar que se recomienda un distanciamiento superior a 25 m (CoFAPyS, 1993), esta situación pone de relieve la posibilidad de contaminación del agua de consumo a través del flujo subterráneo, originada por el vertido de efluentes en pozos absorbentes. 
Se observa una gran diversidad de situaciones y estrategias referidas al uso del recurso. Por ejemplo, un domicilio utiliza el agua de la red pública para el consumo, mientras que aprovecha el agua de pozo para otros usos. Otro caso, muestra que aunque conectados a la red de agua, los propietarios prefieren beber el agua extraída por su bomba de mano, debido a que su sabor le resulta más agradable. Por último, una familia que accede al agua de red y la utiliza para limpieza y preparación de alimentos, prefiere comprar agua de mesa envasada para el consumo familiar debido a las dudas que les genera la calidad del recurso. En dos viviendas particulares no existen cañerías de distribución, por lo cual acarrean el agua desde las bombas manuales hasta el interior de los domicilios. En otras dos viviendas se observaron cañerías externas deficientes.

Las situaciones aludidas ponen de manifiesto la vulnerabilidad de ciertos grupos frente a las interrelaciones entre el uso del recurso subterráneo y la afectación que sobre él producen algunas actividades potencialmente contaminantes. Por un lado, ello obedece al desconocimiento acerca de los riesgos presentes, pero también expresan el convencimiento de los pobladores de que profundizar su perforación o colocar una bomba de mayor capacidad o potencia, permitiría extraer agua de mejor calidad, eliminándose toda situación de riesgo.

\section{b. Hidrodinámica y calidad del agua subterránea}

Entre noviembre de 2011 y agosto de 2013, se efectúo el muestreo sobre 16 perforaciones de una red de medición de los niveles freáticos (Figura 2). Los mapas equipotenciales mostraron que el flujo subterráneo local tiene dirección hacia $\mathrm{N}$ del área de estudio (Figura 3), acorde con el flujo regional (Rodríguez et al., 2013 y Rodríguez, 2014).

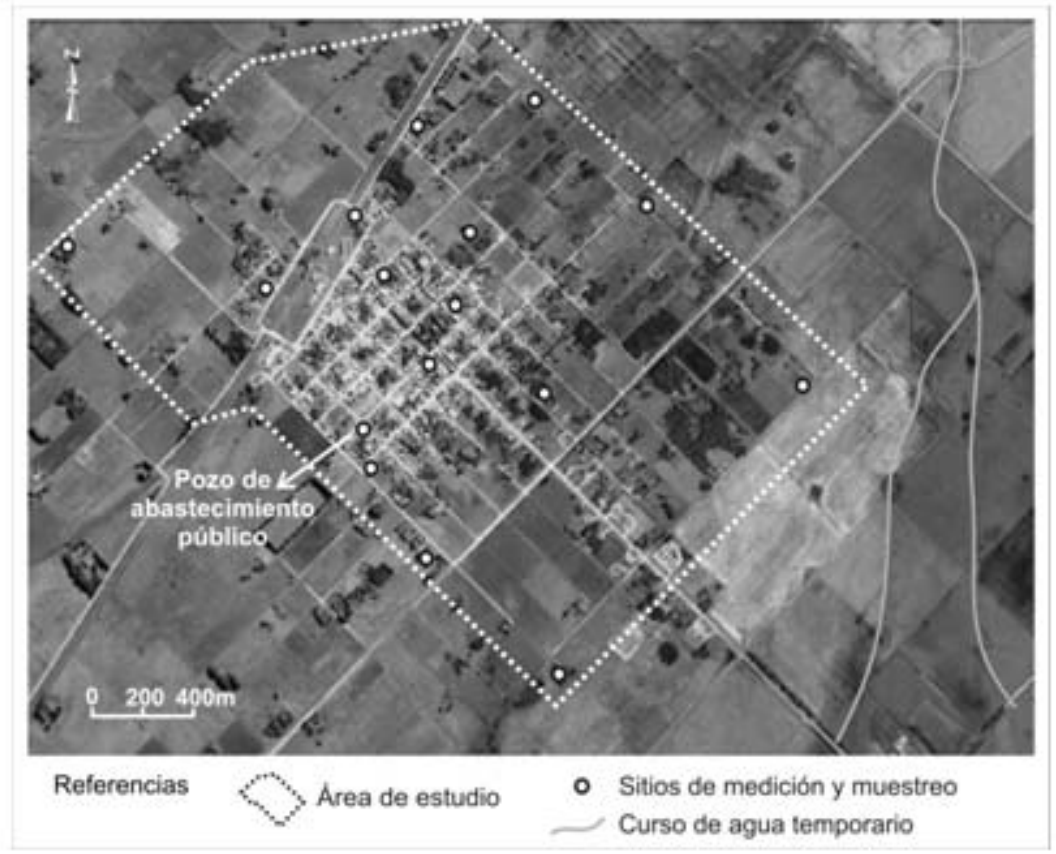

Figura 2. Sitios de medición y muestreo en María Ignacia.

La profundidad del nivel freático en la zona alcanza un máximo de $3 \mathrm{~m}$ en épocas de precipitaciones escasas, condición que origina una elevada vulnerabilidad del acuífero a las cargas contaminantes, hecho especialmente relevante considerando la 
disposición tanto actual como histórica de los efluentes domiciliarios en pozos absorbentes y letrinas. Además, actualmente existen dos situaciones potencialmente contaminantes hacia el acuífero libre, que afectan la calidad del recurso. Una de ellas se relaciona con la localización del cementerio municipal al $S$ de la localidad, ubicado aguas arriba del flujo subterráneo local. La otra se relaciona con la disposición de los residuos sólidos urbanos sin tratamiento en un basural a cielo abierto ubicado al $\mathrm{N}$ de la localidad. Si bien este basural está situado aguas abajo del ejido urbano, constituye un importante foco contaminante del recurso.

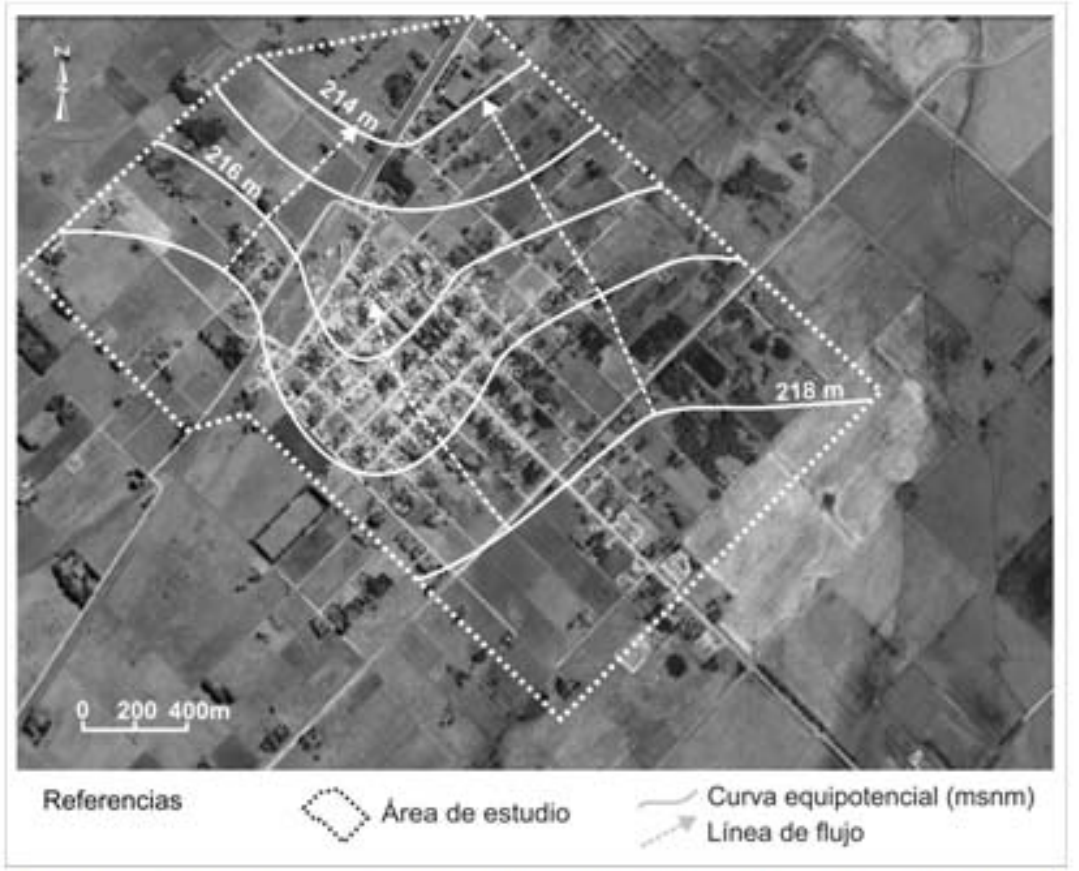

Figura 3. Mapa equipotencial de María Ignacia (09/2012).

La composición predominante del agua subterránea corresponde a bicarbonatada magnésica (Rodríguez et al., 2013). En la Figura 4 se presenta el mapa de curvas de isovalores de conductividad eléctrica para septiembre de 2012, en el cual se observan valores superiores a $900 \mu \mathrm{S} / \mathrm{cm}$ en la mayor parte de la localidad y con un marcado incremento hacia el $\mathrm{N}$, justamente de acuerdo con el sentido de flujo subterráneo. 


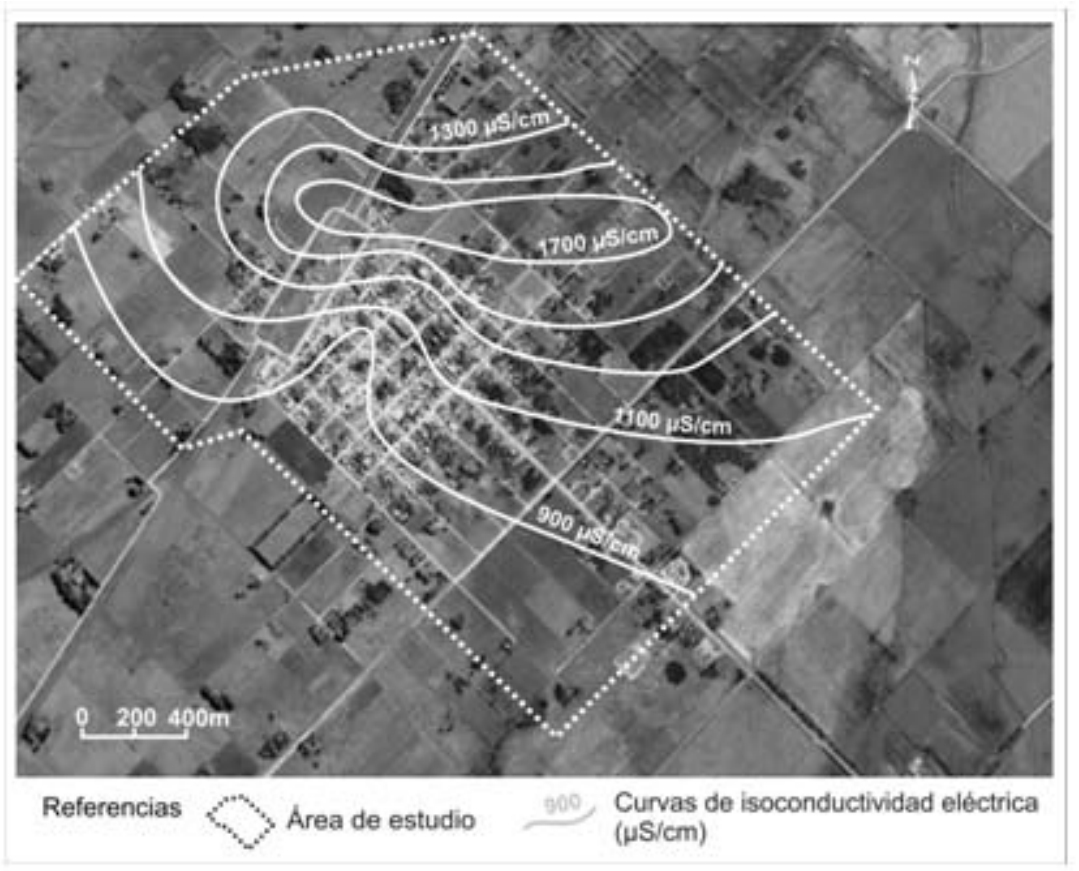

Figura 4. Mapa de isoconductividad eléctrica en María Ignacia (09/2012).

Los nitratos mostraron importantes variaciones, presentando valores entre 12 y 351,7 $\mathrm{mg} / \mathrm{L}$. Las concentraciones de dicho ión señalan el incremento de los valores hacia la zona centro-N (Figura 5), y se atribuyen a las condiciones de las perforaciones, la cercanía de las mismas a los pozos absorbentes y la posibilidad de ingreso de agua por la boca de pozo ${ }^{a}$. 


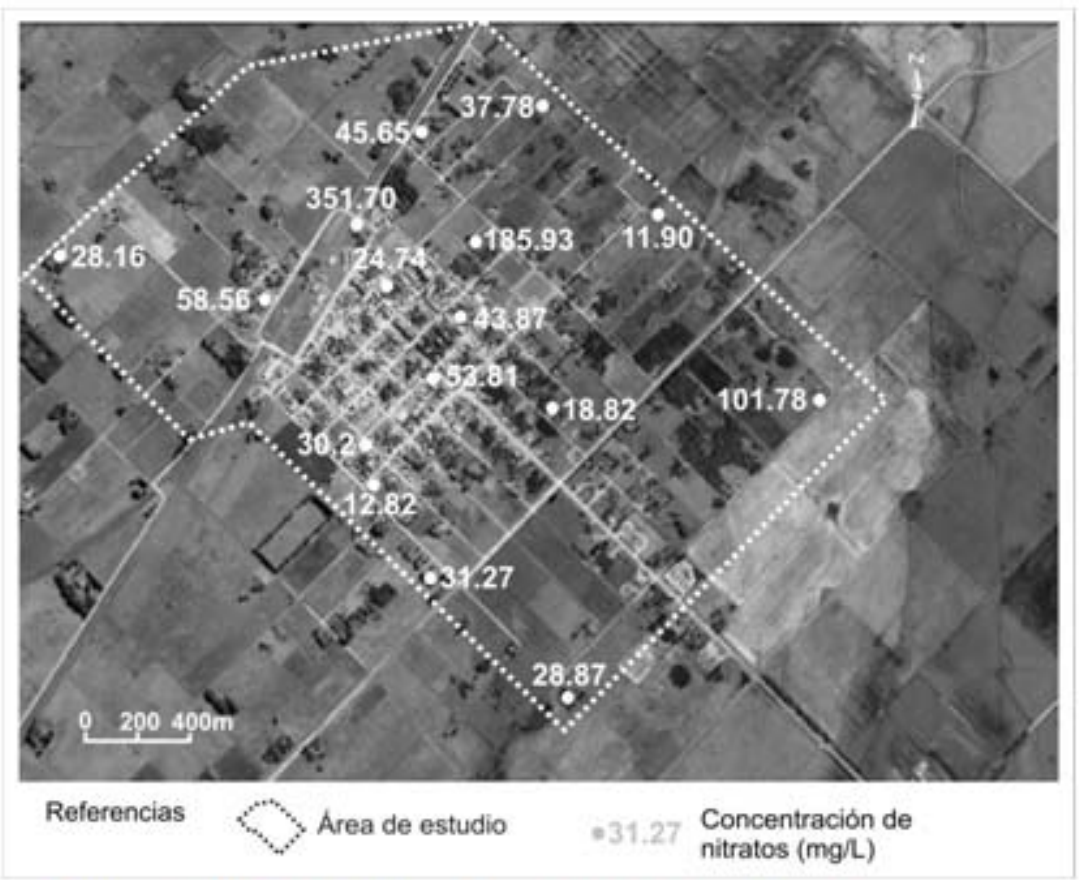

Figura 5. Mapa de concentraciones de nitratos en

$$
\text { María Ignacia (09/2012). }
$$

En relación al nivel máximo de nitratos recomendado por el Código Alimentario Argentino, los muestreos arrojaron entre un $36 \%$ y un $60 \%$ de muestras no aptas para consumo humano, al superar el valor máximo permisible de $45 \mathrm{mg} / \mathrm{L}$ establecido por la legislación argentina (Rodríguez et al., 2013).

Desde el punto de vista microbiológico, entre el $73 \%$ y el $81 \%$ de las muestras resultaron no aptas para el consumo humano según indica el Código Alimentario Argentino (2012). La mayoría de ellas excedió los valores máximos recomendados de coliformes totales y de bacterias aeróbicas mesófilas. Se determinó existencia deEscherichia coli en tres sitios, considerando la peligrosidad que implica la presencia de esta bacteria en aguas de consumo, en tanto no fue detectada Pseudomonas aeruginosa.

La muestra correspondiente al pozo de abastecimiento público de la Cooperativa de Provisión de agua, presentó valores inferiores al máximo recomendado de nitratos y los resultados microbiológicos indicaron su aptitud para consumo humano. Sin embargo, en pozos particulares ubicados en las cercanías (radio de $200 \mathrm{~m}$ ), los contenidos del anión llegaron hasta $70 \mathrm{mg} / \mathrm{L}$.

\section{Gardey}

\section{a. Modos de uso y gestión del agua subterránea}

En Gardey existe cobertura parcial de agua potable por red a cargo de Obras Sanitarias Tandil, ente público dependiente del gobierno municipal. La localidad cuenta con dos perforaciones ubicadas en la planta urbana, cada una de ellas alcanza los 45 m de profundidad, y fueron construidas en 1999 para extraer agua desde el acuífero freático. La red de agua corriente abarca 210 bocas de abastecimiento, que alcanzan un consumo aproximado de entre 60 y $70 \mathrm{~m}^{3} /$ día. 
Una vez extraída el agua a través de las perforaciones municipales, recibe un proceso de desinfección por cloración e ingresa posteriormente a las cañerías de distribución. El ente que provee el servicio realiza controles periódicos de la calidad del agua de la red, no existiendo hasta la actualidad reclamos o problemas vinculados a la calidad del recurso. Sin embargo, el personal técnico encargado de la perforación indicó que no se efectúan controles del contenido de cloro residual existente en las cañerías de distribución.

Simultáneamente a la existencia del sistema de distribución de agua cuya cobertura no alcanza la totalidad del asentamiento, numerosas viviendas se aprovisionan del recurso subterráneo a través de pozos particulares. En primer lugar, porque los usuarios no se encuentran dentro de la zona de tendido de la red municipal, pero además algunos habitantes mantienen en uso sus pozos particulares porque prefieren beber el agua de pozo por motivos organolépticos (especialmente para evitar el consumo de agua con sabor y olor a cloro), o porque el servicio de red experimenta frecuentes interrupciones en el abastecimiento.

El análisis detallado de este conjunto de perforaciones y pozos particulares, indicó predominio de perforaciones someras, y en menor medida de pozos cavados. Para la extracción coexisten en igual medida bombas sumergibles, bombeadores automáticos, bombas manuales y molinos. La mayoría de los pozos de extracción no cuenta con el diseño necesario para su adecuado funcionamiento y para la prevención de la contaminación a través del propio pozo. Las profundidades indicadas por los propietarios varían entre 6 y 48 metros, siendo las perforaciones más profundas las del ente Obras Sanitarias Tandil.

Con respecto a los efluentes domiciliarios, en Gardey no existe sistema de recolección y tratamiento de efluentes cloacales y hasta el momento no se conocen proyectos sobre su futura realización. La disposición de los efluentes líquidos se efectúa principalmente en pozos absorbentes, existiendo también letrinas.

Se relevaron los focos contaminantes dispuestos en las cercanías de las perforaciones y pozos, encontrando que existen pozos absorbentes en su mayoría ubicados a distancias inferiores a 25 metros, valor recomendado por CoFAPyS (1993). Además, se detectaron otros focos como la presencia de animales de cría en cercanías de las perforaciones, la posible afectación por agroquímicos aplicados en zonas agrícolas y una laguna de efluentes de un tambo ubicado a proximidad del asentamiento.

\section{b. Hidrodinámica y calidad del agua subterránea}

Entre septiembre de 2009 y octubre de 2012, se efectúo el muestreo sobre 16 perforaciones de una red de medición de los niveles freáticos (Figura 6). Como se trata de una localidad muy pequeña, se tuvieron en cuenta perforaciones en los alrededores para obtener una mejor representatividad de los resultados. Los mapas equipotenciales (Figura 7) mostraron que el flujo subterráneo local tiene dirección hacia N, acorde a la pendiente regional, aunque se presenta una distorsión hacia el norte de la localidad causada por el menor nivel estático de los pozos de esa zona, es decir, una mayor presión de extracción del recurso (Rodríguez et al., 2011). 


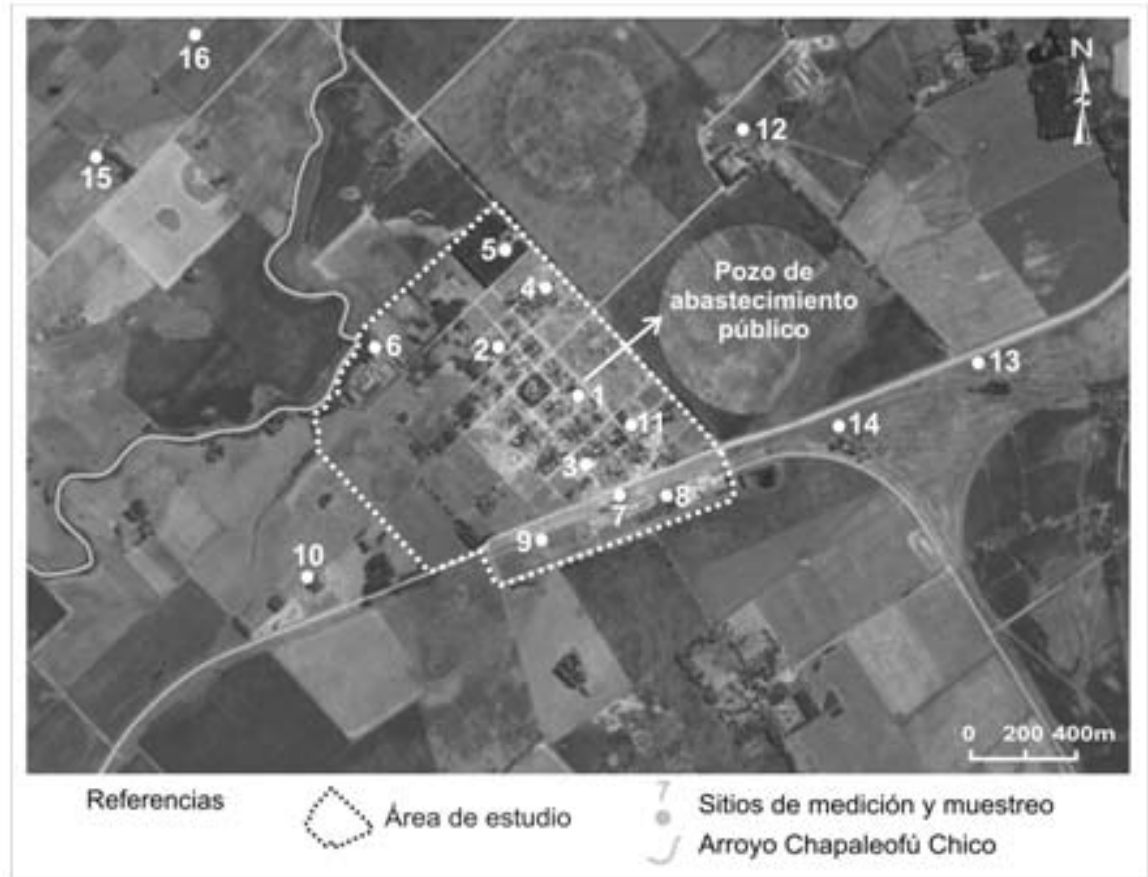

Figura 6. Sitios de medición y muestreo en Gardey.

El nivel freático varía entre 0,5 y 8,6 metros, detectándose las menores profundidades en cercanías del arroyo Chapaleofú Chico y las mayores, en la zona de mayor densidad de pozos de explotación (Figura 7). La escasa profundidad de los niveles freáticos da lugar a una elevada vulnerabilidad del acuífero ante la existencia de cargas contaminantes, especialmente teniendo en cuenta la disposición de efluentes domiciliarios en pozos absorbentes que en muchos casos entran en contacto con la superficie freática. 


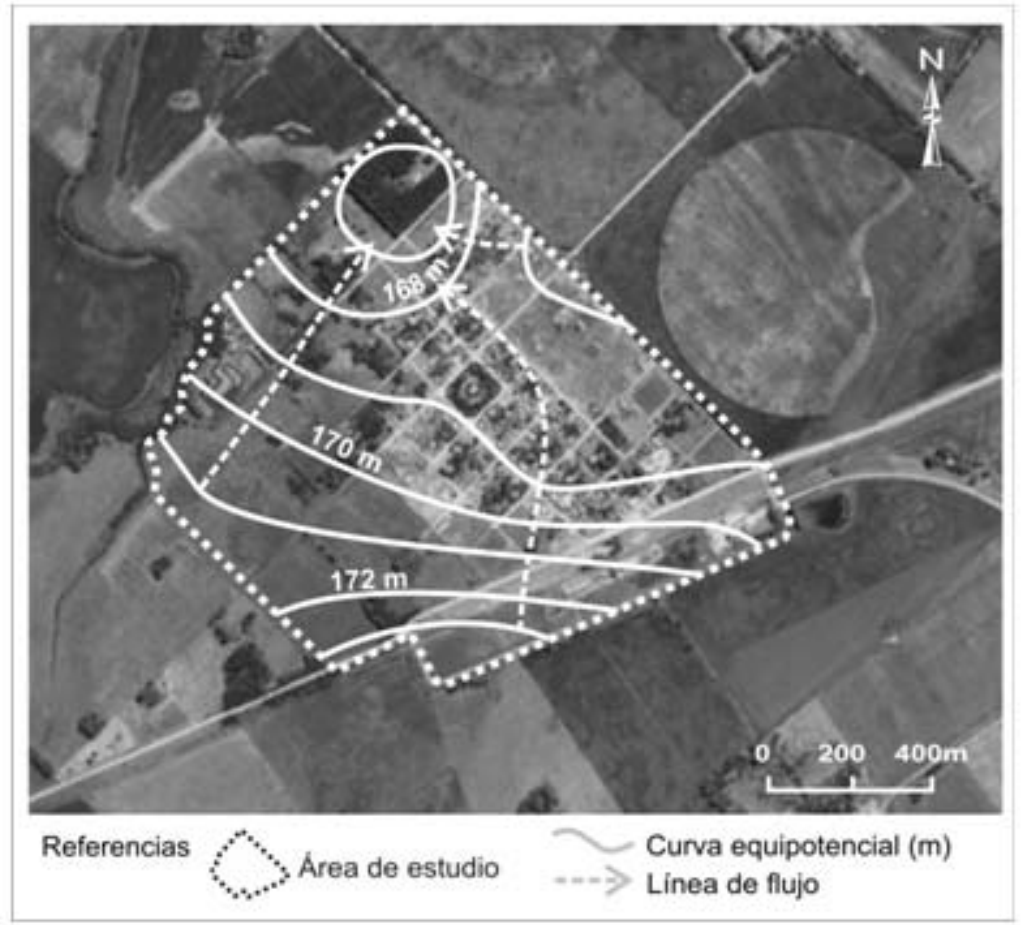

Figura 7. Mapa equipotencial de Gardey (09/2009)

En relación a la calidad del agua, los análisis de iones mayoritarios demuestran una composición bicarbonatada magnésica en su mayor parte (Rodríguez et al., 2011), similar a la detectada en María Ignacia. Ello da a las aguas un carácter joven por su escaso tiempo de permanencia en el acuífero, asociado a la ubicación del sector en la cabecera de cuenca del arroyo Chapaleofú Chico y su proximidad a las zonas serranas de recarga.

Con respecto a la conductividad eléctrica (CE), los valores medios variaron entre 867,92 y $1.014 \mu \mathrm{S} / \mathrm{cm}$ en las distintas oportunidades de medición. El mayor tenor de CE en 2012 puede deberse a la posible contaminación de las aguas subterráneas producida por las elevadas precipitaciones caídas en ese año, lo que generó el ascenso freático y el contacto de la superficie freática con los pozos absorbentes, el desborde de dichos pozos en algunos casos, así como el ingreso de agua y otras sustancias por las bocas de los pozos con inadecuada protección superficial. LaFigura 8 representa el mapa de isoconductividad para septiembre de 2009, donde se observa el incremento de este parámetro hacia el $\mathrm{NE}$, coincidiendo con la zona de mayor concentración de población y por ende de pozos absorbentes. 


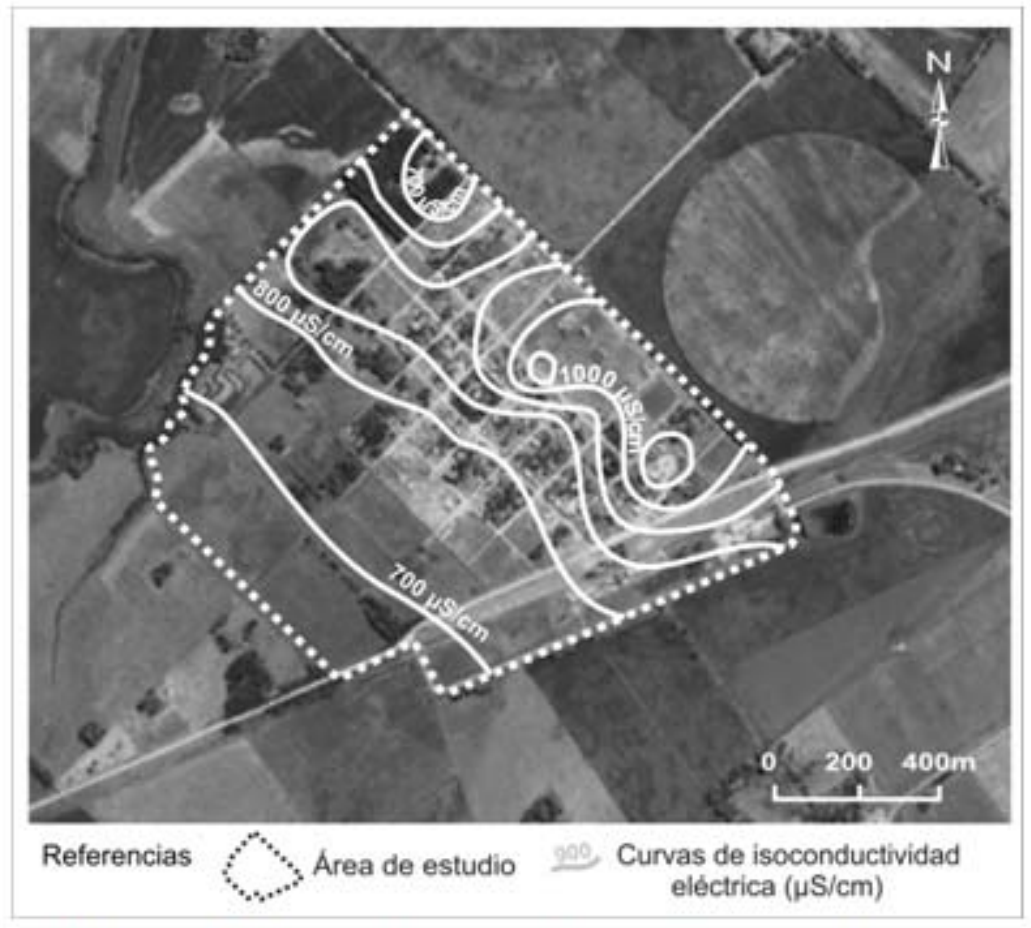

Figura 8. Mapa de isoconductividad eléctrica en

\section{Gardey (09/2009).}

Los valores de nitratos variaron entre 3,5 y $210 \mathrm{mg} / \mathrm{L}$, con excepción de una muestra que superó los $300 \mathrm{mg} / \mathrm{L}$ en dos ocasiones de medición. Los muestreos arrojaron que entre el $38 \%$ y el $58 \%$ de las muestras presentó valores superiores a $45 \mathrm{mg} / \mathrm{L}$, nivel máximo recomendado por el Código Alimentario Argentino (2012) para el consumo humano. En el mapa de isoconcentraciones de nitratos (Figura 9) se observa el incremento de dicho ión en la zona densamente poblada, coincidiendo con el comportamiento de la conductividad eléctrica.

Cabe destacar que el sitio $\mathrm{N}^{0} 1$ corresponde a la perforación para abastecimiento de agua en la red municipal, presentó valores algo superiores a $45 \mathrm{mg} / \mathrm{L}$ de nitratos en cuatro de las cinco campañas, con un máximo de 60,4 mg/L en septiembre de 2009. 


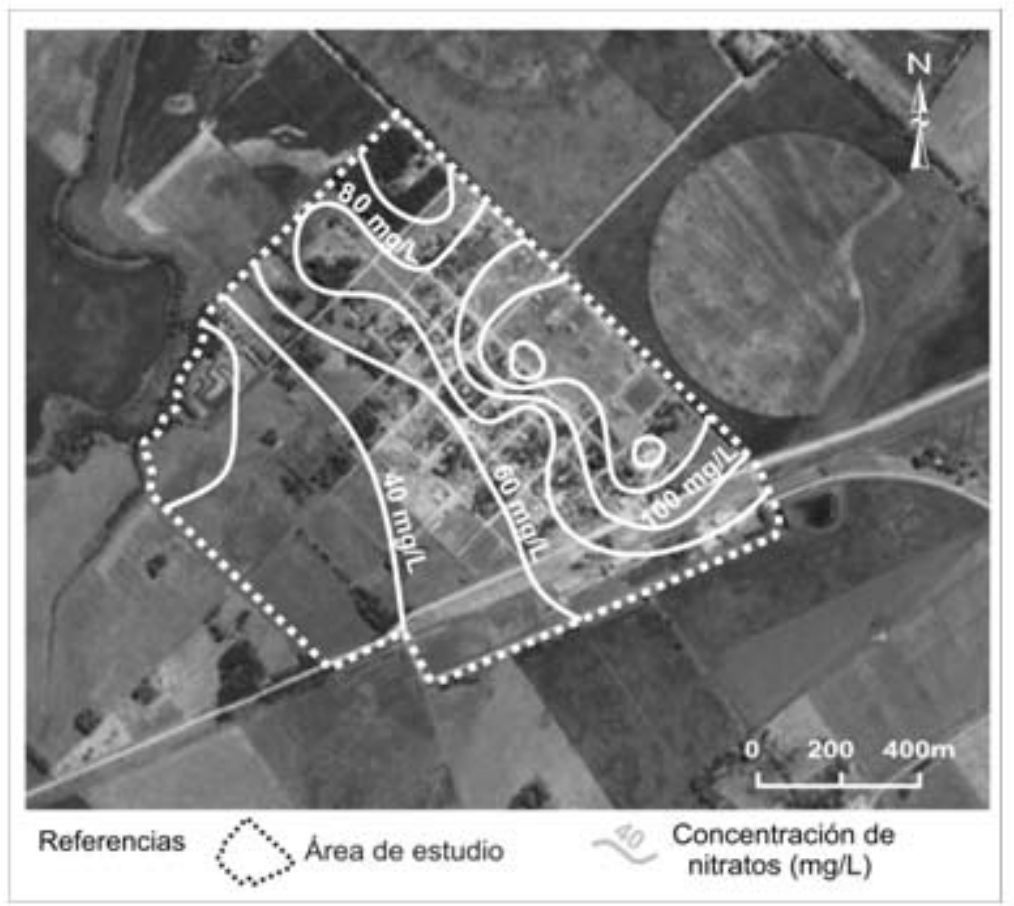

Figura 9. Mapa de concentraciones de nitratos

en Gardey (09/2009).

Respecto a la calidad microbiológica, de las 7 muestras analizadas en 2009 sólo 2 resultaron aptas para consumo humano, siendo una de ellas la de abastecimiento público. Las restantes superaron las recomendaciones del CAA para alguna de las bacterias incluidas. Escherichia coli fue detectada sólo en una muestra, coincidente con los mayores contenidos de nitratos detectados en el ARM y en relación con la presencia de contaminación puntual por disposición de excretas. Por otro lado,Pseudomonas aeruginosa fue hallada en tres sitios (Rodriguez et al., 2011).

El muestreo realizado en 2012, indicó como apta para consumo humano sólo a la muestra correspondiente a la perforación de abastecimiento público. En esta oportunidad, Escherichia coli estuvo presente en cinco muestras y Pseudomonas aeruginosa en una.

Se destaca el alto grado de contaminación del punto $N^{\circ} 4$ que superó todos los parámetros microbiológicos analizados y también fue la de mayor conductividad eléctrica y contenido de nitratos en todas las oportunidades ${ }^{\mathrm{b}}$.

\section{Análisis comparativo entre casos de estudio}

El análisis comparativo de ambos casos (Tabla 1) indica que la problemática hídrica en Gardey y María Ignacia tiene causas comunes. Ellas son determinadas fundamentalmente por la debilidad de la gobernanza local, que se manifiesta principalmente en la cobertura parcial de agua de red, la inexistencia de red cloacal y el escaso conocimiento sobre el funcionamiento del sistema hídrico subterráneo principalmente por parte de la población, que no favorece la protección de la fuente aprovisionamiento de agua. Asimismo, se corrobora que no se cumple la exigencia del Código de Aguas provincial referida a la solicitud de un permiso de perforación para la extracción de agua subterránea por parte de los usuarios particulares. Se suma otra 
debilidad en el caso de María Ignacia dado que el municipio no tiene injerencia sobre la gestión del agua por parte de la cooperativa de servicios.

La conjunción de dichas causas, además del incumplimiento de las normas de prevención de la contaminación entre los sitios de captación y descarga de los efluentes domiciliarios determina un ciclo local de extracción-transporte-disposición cuyo resultado inevitable es la contaminación del agua. Ello se evidencia a través de los contenidos de nitratos y de microorganismos patógenos que exceden lo recomendado por la legislación argentina, especialmente en las perforaciones particulares.

Los problemas detectados en las perforaciones, la presencia de focos contaminantes y el consumo de agua no apta se producen independientemente del nivel socioeconómico de la población. Sin embargo, los sectores de mayores ingresos pueden realizar nuevas y mejores perforaciones así como poseer sistemas de cañerías y tanques de almacenamiento en buen estado, e incluso adquirir agua potable envasada para el consumo humano. 
Tabla 1. Análisis comparativo de los casos de estudio.

\begin{tabular}{|c|c|c|}
\hline & María Ignacia & Gardey \\
\hline $\begin{array}{l}\text { Modos de } \\
\text { abastecimiento } \\
\text { de agua }\end{array}$ & $\begin{array}{l}\text { Una parte de la localidad } \\
\text { posee agua de red a cargo } \\
\text { de una cooperativa. El resto } \\
\text { la extrae del acuífero } \\
\text { mediante pozos particulares, } \\
\text { los cuales no cumplen con el } \\
\text { permiso exigido por ley. }\end{array}$ & $\begin{array}{l}\text { Una parte de la localidad } \\
\text { posee agua de red a cargo del } \\
\text { ente municipal de agua y } \\
\text { saneamiento. El resto la extrae } \\
\text { del acuífero mediante } \\
\text { perforaciones particulares, los } \\
\text { cuales no cumplen con el } \\
\text { permiso exigido por ley. }\end{array}$ \\
\hline $\begin{array}{l}\text { Características } \\
\text { de las } \\
\text { perforaciones } \\
\text { y pozos }\end{array}$ & $\begin{array}{l}2000 \text { habitantes. Población } \\
\text { de nivel socioeconómico } \\
\text { medio y bajo. Predominan } \\
\text { viviendas de uso } \\
\text { permanente, comercios y } \\
\text { actividades vinculadas al } \\
\text { espacio rural. } \\
\text { Adecuado diseño en las } \\
\text { perforaciones de la } \\
\text { cooperativa. Deficiencias en } \\
\text { el diseño y construcción en } \\
\text { las perforaciones } \\
\text { domiciliarias. }\end{array}$ & $\begin{array}{l}640 \text { habitantes. Población de } \\
\text { nivel socioeconómico medio y } \\
\text { bajo. Construcción reciente de } \\
\text { viviendas de clase media-alta. } \\
\text { Predominan viviendas de uso } \\
\text { permanente, comercios y } \\
\text { actividades vinculadas al } \\
\text { espacio rural. } \\
\text { Adecuado diseño en las } \\
\text { perforaciones municipales. } \\
\text { Deficiencias en el diseño y } \\
\text { construcción en las } \\
\text { perforaciones domiciliarias. }\end{array}$ \\
\hline $\begin{array}{l}\text { Focos } \\
\text { contaminantes }\end{array}$ & $\begin{array}{l}\text { Pozos absorbentes. } \\
\text { Criaderos de animales. } \\
\text { Basural a cielo abierto. } \\
\text { Cementerio. }\end{array}$ & $\begin{array}{l}\text { Pozos absorbentes. Tambo. } \\
\text { Planta de fabricación de } \\
\text { agroquímicos. }\end{array}$ \\
\hline $\begin{array}{l}\text { Disponibilidad } \\
\text { de agua }\end{array}$ & $\begin{array}{l}\text { No se presentan problemas } \\
\text { de disponibilidad/cantidad de } \\
\text { agua. Las autoridades de la } \\
\text { cooperativa atribuyen un } \\
\text { descenso de niveles freáticos } \\
\text { a la aplicación de riego en el } \\
\text { cultivo de papa realizado en } \\
\text { la zona. }\end{array}$ & $\begin{array}{l}\text { No se presentan problemas de } \\
\text { disponibilidad o cantidad de } \\
\text { agua. }\end{array}$ \\
\hline
\end{tabular}




\begin{tabular}{|c|c|c|}
\hline $\begin{array}{l}\text { Calidad del } \\
\text { agua }\end{array}$ & $\begin{array}{l}\text { El agua de red resultó apta } \\
\text { para consumo humano. } \\
\text { Problemas de calidad } \\
\text { química y microbiológica en } \\
\text { pozos particulares. }\end{array}$ & $\begin{array}{l}\text { El agua de red supera el valor } \\
\text { máximo recomendado de } \\
\text { nitratos para consumo } \\
\text { humano. Problemas de calidad } \\
\text { química y microbiológica en } \\
\text { pozos particulares. }\end{array}$ \\
\hline $\begin{array}{l}\text { Sistema } \\
\text { cloacal }\end{array}$ & $\begin{array}{l}\text { No posee sistema de } \\
\text { recolección cloacal. Existe un } \\
\text { proyecto de tendido cloacal } \\
\text { aprobado, con dificultades } \\
\text { para su inicio. }\end{array}$ & $\begin{array}{l}\text { No posee sistema de } \\
\text { recolección cloacal y no se } \\
\text { conocen proyectos al respecto. }\end{array}$ \\
\hline $\begin{array}{l}\text { Gestión local } \\
\text { del agua }\end{array}$ & $\begin{array}{l}\text { La cooperativa de servicios } \\
\text { provee el agua potable para } \\
\text { el abastecimiento y recibe } \\
\text { controles del organismo } \\
\text { provincial responsable del } \\
\text { agua en el ámbito rural. El } \\
\text { municipio no tiene injerencia } \\
\text { en la gestión del agua. } \\
\text { El proyecto de sistema } \\
\text { cloacal es responsabilidad } \\
\text { del gobierno provincial. }\end{array}$ & $\begin{array}{l}\text { El abastecimiento de agua se } \\
\text { coordina a través del municipio } \\
\text { desde la ciudad cabecera del } \\
\text { distrito (Tandil), sin injerencia } \\
\text { de la población local. No se } \\
\text { realiza planificación de la } \\
\text { provisión del recurso a medida } \\
\text { que se produce crecimiento } \\
\text { poblacional en la localidad. }\end{array}$ \\
\hline
\end{tabular}

\section{CONCLUSIONES}

La expansión de funciones residenciales, los nuevos usos turísticos y la valorización de las amenidades ambientales ponen de manifiesto las transformaciones experimentadas por los pueblos rurales pampeanos en la última década. A los requerimientos de agua y a los impactos sobre el recurso impuestos por la intensificación agrícola, se suman las demandas en cantidad y calidad exigidas por la población para el consumo y el desarrollo de actividades productivas, lo que interpela sobre la necesidad de interpretar estos procesos de cambio territorial a la luz de la presión que producen sobre la demanda y uso de los recursos hídricos.

La disponibilidad de agua y la gestión de los recursos hídricos constituyen ejes centrales para el desarrollo de los espacios rurales. El abordaje realizado en este trabajo a través de los estudios de caso da cuenta de la sensibilidad de los sistemas hídricos frente a las demandas planteadas. La gestión sustentable de los recursos hídricos en los espacios rurales debiera contemplar la interdependencia de los sistemas, la fragilidad de los equilibrios naturales, los impactos de las actividades sobre el estado de los recursos y fundamentalmente la armonización de acciones de los usuarios (Ardillier-Carras, 1997).

Ante todo lo expuesto, se considera necesaria y fundamental la creación de capacidades locales de gobernanza, de modo que los pueblos rurales tengan injerencia en las decisiones sobre el manejo de los recursos hídricos a través de la participación de actores propios. Como sustento para las decisiones, se requiere el monitoreo continuo del estado de los recursos hídricos y sus potenciales impactos, así como la comunicación de la información relevada y su apropiación por parte de los actores involucrados. Estos procesos de participación e información deben ser acompañados por estrategias educativas y de capacitación sobre los aspectos hidrológicos generales, sobre la importancia de los recursos hídricos en el sistema ambiental y sobre el rol estratégico que ellos juegan en el desarrollo de los territorios rurales. 


\section{REFERENCIAS}

- Ardillier-Carras, F. (1997). L'eau, ressource pour le développement d'un espace rural. Presses Universitaires de Limoges.

- CoFAPyS - Consejo Federal de agua potable y saneamiento (1993). Normas de estudio, criterios de diseño y presentación de proyectos de desagües cloacales para localidades de hasta $\mathbf{3 0 . 0 0 0}$ habitantes. Fundamentación de normas. II: 7. Argentina.

- Jacinto, G. (2011). Asentamientos de rango menor (ARM) en Tandil: transformaciones territoriales a partir de la renovación de los vínculos urbano-rurales.Estudios Socioterritoriales. Revista de Geografía, № 10, pp. 103-124.

- Jacinto, G.; Nogar, M. L.; Nogar, A. G.; Clementi, L. (2014). Yuxtaposión de actores y de lógicas en asentamientos de rango menor de la pampa argentina. En: Valenzuela, C.; García, A.; Rosa, P. (comp.) Inclusión social en las economías regionales. Estrategias participativas y propuestas de articulación social en el territorio. CEUR CONICET IIGHI CONICET UNNE, Resistencia, pp. 73-96.

- Municipalidad de Tandil (2005). Plan de Desarrollo Territorial. Tandil.

- Perrier-Cornet, Ph. (Dir.) (2002). A qui appartient l'espace rural? Enjeux publics et politiques. Paris: Editions de l'Aube.

- Rodríguez, C. I.; Miguel, R. E.; André, M. J.; García, M. C.; Ruiz de Galarreta, A.; Banda Noriega, R. (2011). Análisis del agua subterránea en la localidad de Gardey (Tandil, Buenos Aires). En: Calidad y contaminación del agua subterránea. Actas del VII Congreso Argentino de Hidrogeología, pp. 132-139. Salta.

- Rodríguez, C. I.; Díaz, A.; Jacinto, G.; Tabera, A.; Pessolano, B.; Ruiz de Galarreta, A. (2013). Evaluación del agua subterránea y transformaciones territoriales en María Ignacia-Vela, Tandil (Buenos Aires, Argentina). En: Agua subterránea recurso estratégico. Actas del VIII Congreso Argentino de Hidrogeología, Tomo I, 306-313. Editorial EDULP, La Plata. 
- Rodríguez, C. I. (2014). Evaluación ambiental del uso y gestión del agua subterránea en el partido de Tandil. Pautas para su gestión sustentable. Universidad Nacional de La Plata, La Plata.

- Sili, M. (2005). La Argentina rural. De la crisis de la modernización agraria a la construcción de un nuevo paradigma de desarrollo de los territorios rurales, Instituto Nacional de Tecnología Agropecuaria (INTA), Buenos Aires.

- Sili, M. (2010). ¿Cómo revertir el proceso de fragmentación de los territorios rurales? Instituto Nacional de Tecnología Agropecuaria (INTA). Buenos Aires.

- Vapñarsky, C. y Gorojovsky, N. (1990) El crecimiento urbano en la Argentina. Grupo Editor Latinoamericano, Buenos Aires.

- Villalba, M. S. (2015). Determinación de usos del suelo como herramienta para la gestión ambiental de asentamientos de rango menor. Gardey (Partido de Tandil). Facultad de Ciencias Humanas, Universidad Nacional del Centro de la Provincia de Buenos Aires, Tandil.

1. Licenciada en Diagnóstico y Gestión Ambiental. Magíster en Ecohidrología. Doctora en Ciencias Naturales. Consejo Nacional de Investigaciones Científicas y Técnicas (CONICET). Centro de Investigaciones y Estudios Ambientales (CINEA), Universidad Nacional del Centro de la Provincia de Buenos Aires, Argentina.corodri@fch.unicen.edu.ar ORCID ID 0000-0001-5596-5035.

2. Profesora de Geografía. Magister Scientiae en Gestión Ambiental del Desarrollo Urbano. Doctora en Geografía-Ordenamiento del Territorio-Urbanismo. Consejo Nacional de Investigaciones Científicas y Técnicas (CONICET). Centro de Estudios Sociales de América Latina (CESAL), Universidad Nacional del Centro de la Provincia de Buenos Aires, Argentina.guillermina.jacinto@gmail.com ORCID ID 0000-00024352-2699

a. El mapa de concentraciones de nitratos se elaboró con puntos que muestran sólo los valores, considerando ese modo el más apropiado, en lugar de curvas de isoconcentraciones, ya que éstas se verían distorsionadas ante la presencia de valores muy disímiles entre sitios de muestreo ubicados a corta distancia.

b. Los residentes de la vivienda correspondiente comenzaron a consumir agua de red a partir de las recomendaciones brindadas, ante los resultados contundentes obtenidos en los distintos análisis de agua subterránea. 
Para citar este artículo: Rodríguez, C.I. y Jacinto, G.P. (2017). Recursos hídricos: calidad y proceso de gestión en la región pampeana argentina. Luna Azul, 44, 59-79. DOI: $\quad 10.17151 / l u a z .2017 .44 .5, \quad$ Recuperado de http://200.21.104.25/lunazul/index.php?option=com_content\&view=article\&id=220

Esta obra está bajo una Licencia de Creative Commons Reconocimiento CC BY

(). (1)

